\title{
Research on The Emergency Decision Support System of Unexpected Events Based on Case-based Reasoning
}

\author{
Xing-Hua $L^{1, a}$ and Zhi-Xin MA ${ }^{1, b, *}$ \\ ${ }^{1}$ Army Engineering University-Shijiazhuang Campus, China \\ a lu-xh163@163.com , b 907229834@qq.com \\ ${ }^{\star}$ Corresponding author
}

\begin{abstract}
Keywords: Unexpected Events, Case-Based Reasoning, Emergency Decision Support System, Emergency Preplan.
\end{abstract}

\begin{abstract}
According to the characteristics of emergency events, in order to improve the efficiency of emergency decision, Combining decision support system and CBR, this paper constructs the system structure of emergency decision support system based on case-based reasoning, and studies the generation method and reasoning mechanism of emergency preplan.
\end{abstract}

\section{Introduction}

In recent years, with the rapid development of social economy, the acceleration of industrialization and the wide application of new technology in many fields, the overall speed of social progress has been greatly improved. At the same time, the number of social public security unexpected events is rising, and the destructiveness is increasing. Especially the spread of international terrorist forces not only cause frequent social emergencies, but also bring great harm to people's life and property safety.

The unexpected events have the characteristics of abrupt, seriousness and urgency, in the face of these events, due to the limited rationality of human beings, if people make decisions only by brain and experience, the human has been difficult to meet emergency requirements, must use the modern scientific and technological means to help decision makers make decisions, improve the efficiency and effectiveness of decision making. Quick response to unexpected events is made to reduce the adverse effects and losses caused by events, this is the problem of emergency decision making. Emergency decision is a non-programmed decision problem, we can play the advantage of decision support system in dealing with semi-structured and unstructured problems, and develop an emergency decision support system(EDSS) for supporting emergency decision-making, this can enhance effectiveness of emergency decision making, can save valuable time to improve the efficiency of decision making. At the same time, case-based reasoning (CBR) in artificial intelligence can be used to reasoning through previous experience and solving knowledge of similar problems, make corresponding adjustment according to the difference between old and new situation, thus the solution of the new problem is obtained and a new case is added to the case base. Through the self-learning behavior of EDSS, the number and quality of cases in the case base will increase, and the experience of EDSS will become more and more abundant, and the effect of assistant decision will also get better and better. This paper attempts to combine DSS and CBR technology to construct the EDSS for the emergency, EDSS can make full use of stored data, model, case and knowledge, and the use of advanced system analysis tools, and the high speed computation and reasoning function, information processing ability, through human-computer interaction, to extend the rational ability of decision-makers, to enhance psychological endurance of decision-makers, So that the decision-makers can mainly focus on the use of their experience, intuition and insight of judgement and choice of key issues, so as to improve the scientificity and effectiveness of emergency decision making $^{[1,2]}$. 


\section{Case based Emergency Decision Support Method}

Case based emergency decision support method can be divided into three stages.

\section{Expression of Emergency Case}

The expression of emergency case is mainly to collect representative emergency cases, and to reorganize and extract the key content and input the system. According to the research results of current scholars, the expression of emergency case is mainly about the general information about events, such as the time, place and number of casualties. There are two problems in this approach: one is that it does not reflect the process of the event, because emergency cases describe the complete process of an event (including occurrence, development, control and elimination), which includes time characteristics, it is not comprehensive to describe only the use of the general situation; two is that there is no record of the disposal process at the time, because we want to record not only what happened, but also how to deal with it, even including the evaluation of the effect of each disposal instruction(a simple classification can be used to distinguish between correct and wrong ways of handling).

One more question needs to be considered. At present, most scholars manage unexpected events as single objects, but in reality, there are many secondary events after a starting and major event occurs, for example, flood disasters can cause plague, public safety incidents may cause traffic problems, and so on. There are also great differences in the nature of these events, so they need to take their respective measures. This is the main obstacle in the current emergency decision making: for emergency decision-making of multi event symbiosis and complex situation, multiple events in this state are mutual decision making backgrounds, or the same events may need different disposal methods under different backgrounds ${ }^{[3]}$.

Therefore, the expression of emergency case can be improved by two points:

(1) The process characteristics of events: recording according to the disposal process, mainly including the status of events on some main time nodes, the measures taken and the execution results.

(2) The background characteristics of events: when recording a case, it is necessary to analyze the relationship between the various secondary events that occur during this process, and it needs to be reflected in some way.

\section{Searching of Emergency Case}

Searching for similar cases that have happened in case base, most scholars search for similar cases based on simple attributes of events, such as time, place and level. According to the description of the emergency case, we should be able to get more accurate search results, mainly from two aspects:

Searching according to the event background--that is the state of the surrounding environment when the event occurs, or other events that are happening at the same time, if the similarity of the background of the two events (Composed of multiple events) is higher, then the similarity of the two events is also higher ${ }^{[4]}$.

Searching according to event development--that is the development trend of the event itself, without considering the surrounding environment. If the attributes of the two events are closer in their development process and speed, the similarity of these two events is relatively high.

\section{The Generation of Emergency Preplan}

The basic thinking of generating emergency preplan based on case base is: first of all, to find the high similarity of the case (see previous step), then the disposal measures taken in these cases are displayed according to time order, effect classification and subordinate relationship of events or secondary events, which is as a reference for the decision-maker, and in the end it is directly adopted or adjusted to be decided by the decision-maker. 
An emergency preplan is a sequence composed of multiple instructions. Specifically, each instruction can be described in terms of publisher, release time, receiver, instruction content, subordinate event (possibly a secondary event), consumption of resources, execution effect and so on. If the second step search gets multiple similar cases, it is also possible to highlight the disposal measures used in multiple cases according to the frequency of their occurrence ${ }^{[5]}$.

\section{The Architecture of the EDSS}

The architecture of the EDSS is shown in Figure 1.

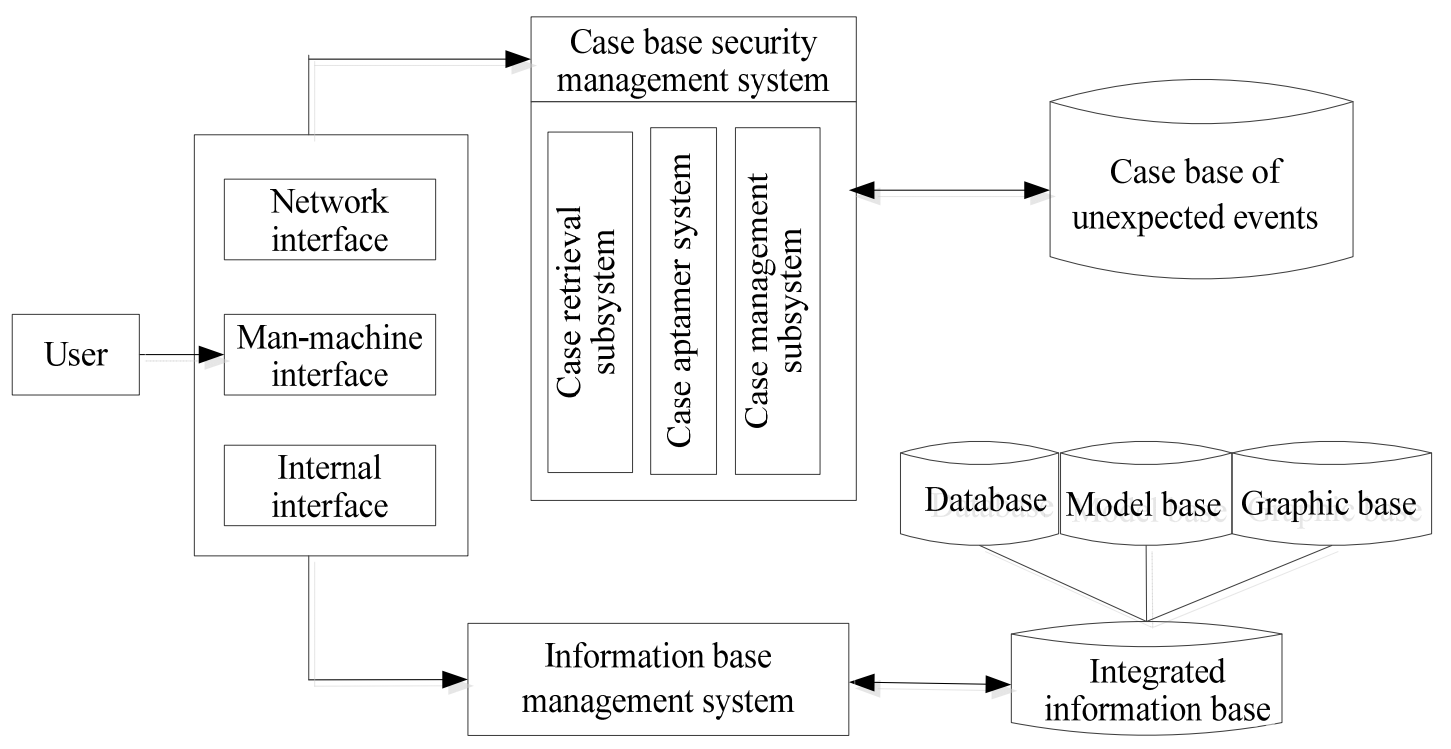

Figure 1. Architecture of EDSS for unexpected events

\section{Interface System}

The interface system consists of three parts: man-machine interface, network interface and internal interface. The human-computer interface is mainly to establish the interaction between the user and the system, including the user input equipment emergency information and the system output information. In most cases, the information that users grasp is limited, and most of them are low-level information such as fuzzy information, uncertain information and incomplete information, which requires human machine interface to have strong ability of information recognition and processing. Man-machine interface can be human-computer interaction through heuristic induction, multiple selection, problem filling, non-question and answer, parameter default and so on. The basic function of network interface is to connect user application and network communication program. On the one hand, users can exchange information with remote nodes better, and on the other hand, it can realize resource sharing of multiple systems. The internal interface is used for the connection between the main system and other software systems.

\section{Case base of Unexpected Events and its Management System}

The case base of unexpected events store all kinds of emergencies, that is, a complete and true description of the past emergencies, solutions and their implementation results. Among the solutions about unexpected events, both successful experience, do not rule out the possibility of failure, so that the decision makers will be able to in the new situation, draw two and knowledge of emergency information to make more reasonable judgment, and have a more effective decision. At the same time, cases in emergencies case library must be diversified and timeliness, so that it can provide a valuable reference case for emergency decision-making in complex crisis scenarios. 
The case base management system is composed of three subsystems: case retrieval, case adaptation and case management. The case retrieval subsystem is responsible for interpreting the user's search request, to complete the retrieval of the case and plan specific case; case adaptation subsystem is responsible for the retrieved source case is modified, so that it can meet the requirements of solving the current problems; case management subsystem is responsible for the increase, delete and modify the operation of the crisis case base.

\section{Comprehensive Information base and its Management System}

Comprehensive information base is actually a multidatabase system, including database, model base and graphics base. The information stored in the comprehensive information base includes the constrained constraints dependent on the man-machine interface, rules, knowledge, technical indicators, and all kinds of emergency information ( such as human, material and financial resources, etc.), and all kinds of reasoning rules, algorithms and functions based on case-based reasoning. The information base management system performs operations on all kinds of information bases, and realizes the consistency of the comprehensive information base. It includes the corresponding database management system, the model base management system and the graphics case management system.

\section{Reasoning Mechanism based on the Emergency Preplan for Unexpected Events} 2.

The process of reasoning based on the emergency preplan of unexpected events is shown in Figure

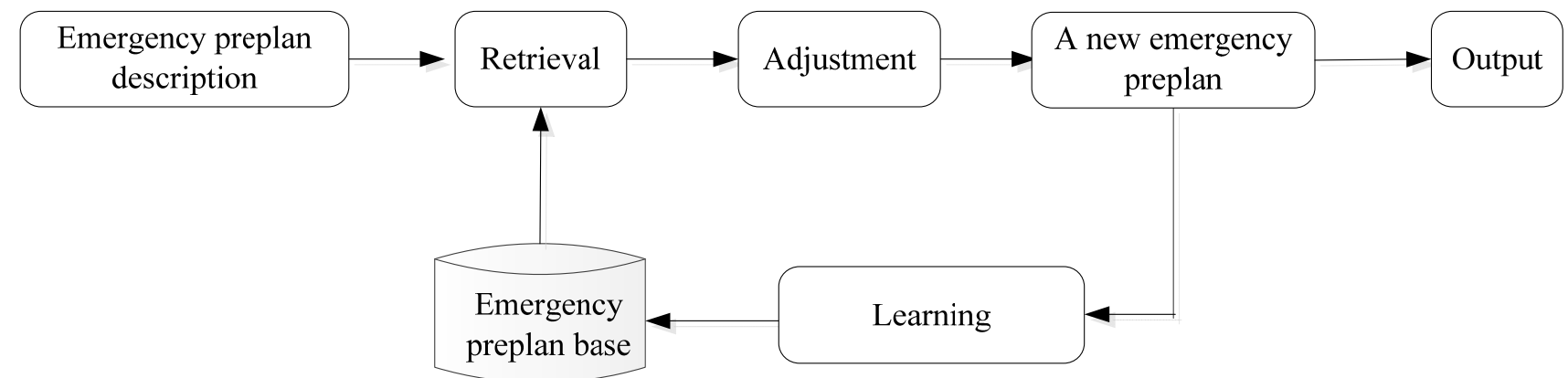

Figure 2. The process of Preplan reasoning

\section{Preplan Description}

The preplan description is to express the preplan as the information that the computer system can store and identify. A typical the emergency preplan of unexpected events usually consists of three parts: (1) description of crisis scene: problems to be solved when the crisis occurs and the surrounding environment; (2) Emergency plan: a solution to an unexpected events; (3) Results: results after implementation of the plan. There are many ways of presentation for preplan, such as semantic network, framework, decision tree, prototype, neural-network and object-oriented technology. The presentation of the preplan should be able to show all kinds of knowledge needed to solve the problem in a more comprehensive way, and have a good modular structure to facilitate the maintenance of the plan bank. Therefore, we use an object-oriented framework based approach to represent the case, as shown in Table 1.

The method encapsulates factual knowledge, commonsense knowledge, experiential knowledge, control knowledge and process knowledge in relatively independent preplan objects, which is clear level, flexible structure and strong adaptability, and conducive to the organization, management and maintenance of the preplan base. 
Table 1. Framework structure of the preplan

Preplan: $=$ the actual plan template: $=<$ attribute list, solutions [, list of sub-plans] [, the list of actual plans];

Attributes list: $=$ attribute 1 [, attribute $2[$, attribute $3[\ldots]]]$

Attribute: $=$ number, words, symbols, or other types of digit;

Sub-preplan list: = sub-preplan 1 [, sub-preplan 2 [, sub-preplan $3[\ldots]]$;

Sub-preplan: $=$ the actual plan pointer;

The actual plan list: $=$ the actual plan pointer 1 [, the actual plan pointer 2 [, the actual plan pointer $3[\ldots]]]$;

Solution: $=$ a structured representation of the sequence, judgment, loop and concurrency structure with sub-preplan sign;

Solution process: = structured words description using attributes or not using attributes;

Actual case: $=<$ case pointer, attributes list, solution process, evaluation [, sub-cases list] $>$;

Case pointer: $=$ the only sign of the case used in this case;

Evaluation: $=$ the text description, score;

Evaluation: $=$ a quantitative score or a description of a quantifiable grade.

\section{Preplan Retrieval}

The goal of preplan retrieval is to retrieve a group of similar plans, according to the definition and description of the new problem, which are as few as possible, and have a reference meaning for solving problems, and is as the basis for solving new problems. The common preplan retrieval algorithms include the nearest neighbor strategy, the inductive reasoning strategy, the knowledge guidance strategy and the template retrieval strategy.

The process of preplan retrieval is: according to problem description information by the input of the decision-maker, the feature information of the decision problem is obtained through the user interface; then according to the index information of the feature information base, it is retrieved in the feature database to obtain the preplan feature information related to the feature information of the decision problem; and then according to the feature information of the preplan, the preplan content that corresponds to it can be obtained in the preplan base. If a new preplan is added to the preplan base, the feature data indexer gets the feature information of the new preplan and puts it into the The process of preplan retrieval is: according to problem description information by the input of the decision-maker, the feature information of the decision problem is obtained through the user interface; then according to the index information of the feature information base, it is retrieved in the feature database to obtain the preplan feature information related to the feature information of the decision problem; and then according to the feature information of the preplan, the preplan content that corresponds to it can be obtained in the preplan base. If a new preplan is added to the preplan base, the feature data indexer gets the feature information of the new preplan and puts it into the feature database, when the useless preplan is deleted, the feature information corresponding to the preplan can be deleted in the feature database, and the retrieval process is shown in Figure 3. 


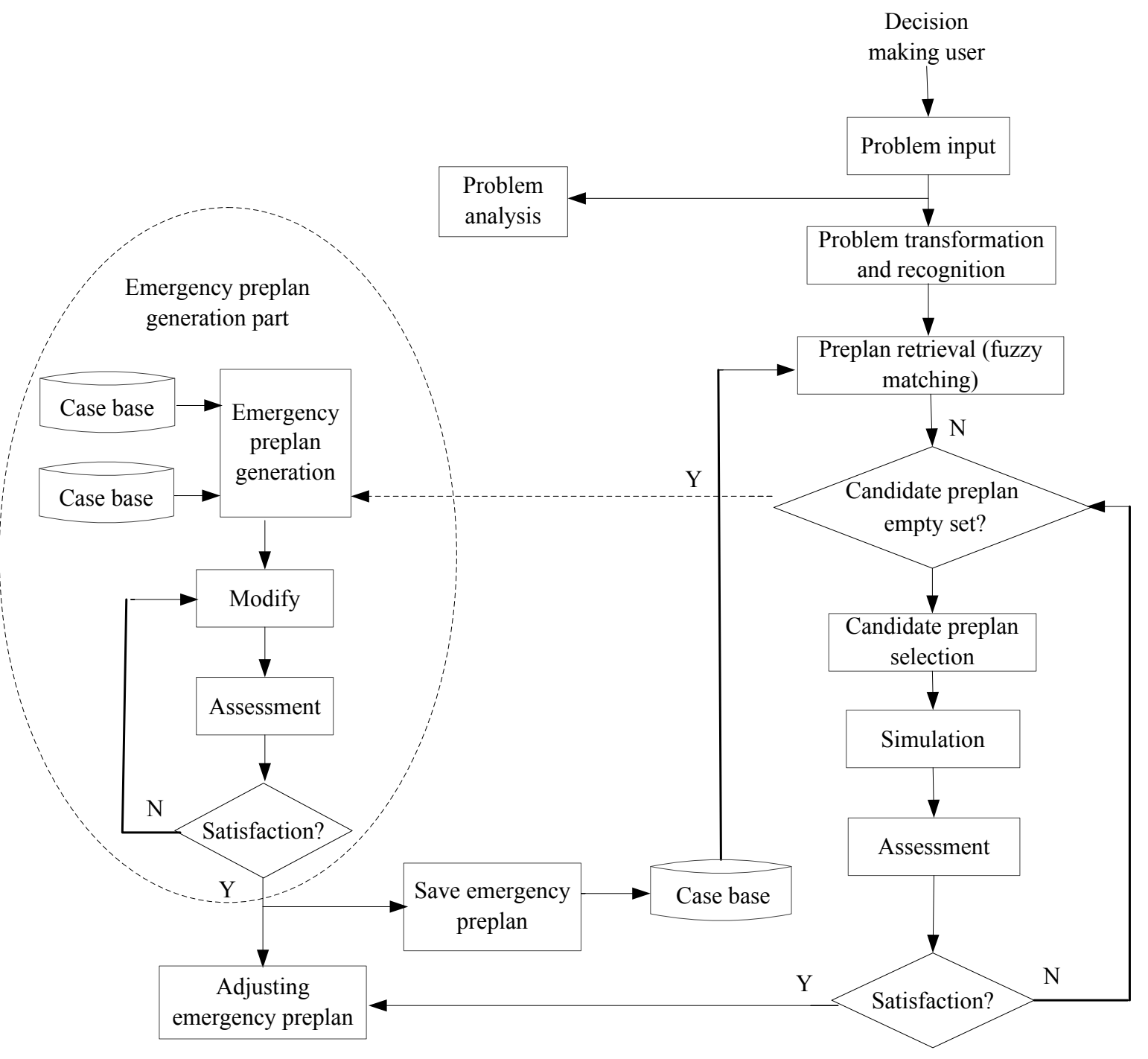

Figure 3. The retrieval process of the preplan

\section{Adjustment of the Preplan}

A preplan retrieved from the source preplan base is similar to the current problem, but there will be a certain difference between the two and it is difficult to match completely. Therefore, it is necessary to adjust preplans. Preplan adjustment is based on the domain knowledge and experience of the knowledge base as a guide, according to the difference between the old and new preplans, people are going to adjust, repair and integrate the solving method of the retrieved preplans, so as to compensate for those inconsistent places and make the adjusted solution plan for the current problem, so as to get the solution of the current problem.

When the source preplan is close enough to the current problem, and the source solution meets the requirements of the current problem, it can't be adjusted, and directly use the retrieved source preplan as a solution to the current problem. However, in most cases, especially when the system experience is not enough, the source preplan can't fully meet the requirements of the current problem, so we need to adjust the preplan. The transformational adjustment strategy and the parameter adjustment strategy are usually adopted in the pre plan adjustment. Their basic idea is to compare the differences between the retrieved preplans and the attributes of the new problem, and to adjust the scheme to the right direction. There are generally several forms of adjustment: adding new contents to old solutions, cutting out some contents from old solutions, replacing some contents in old solutions, and reconverting some parts of old solutions. The adjustment process is shown in Figure 4. 


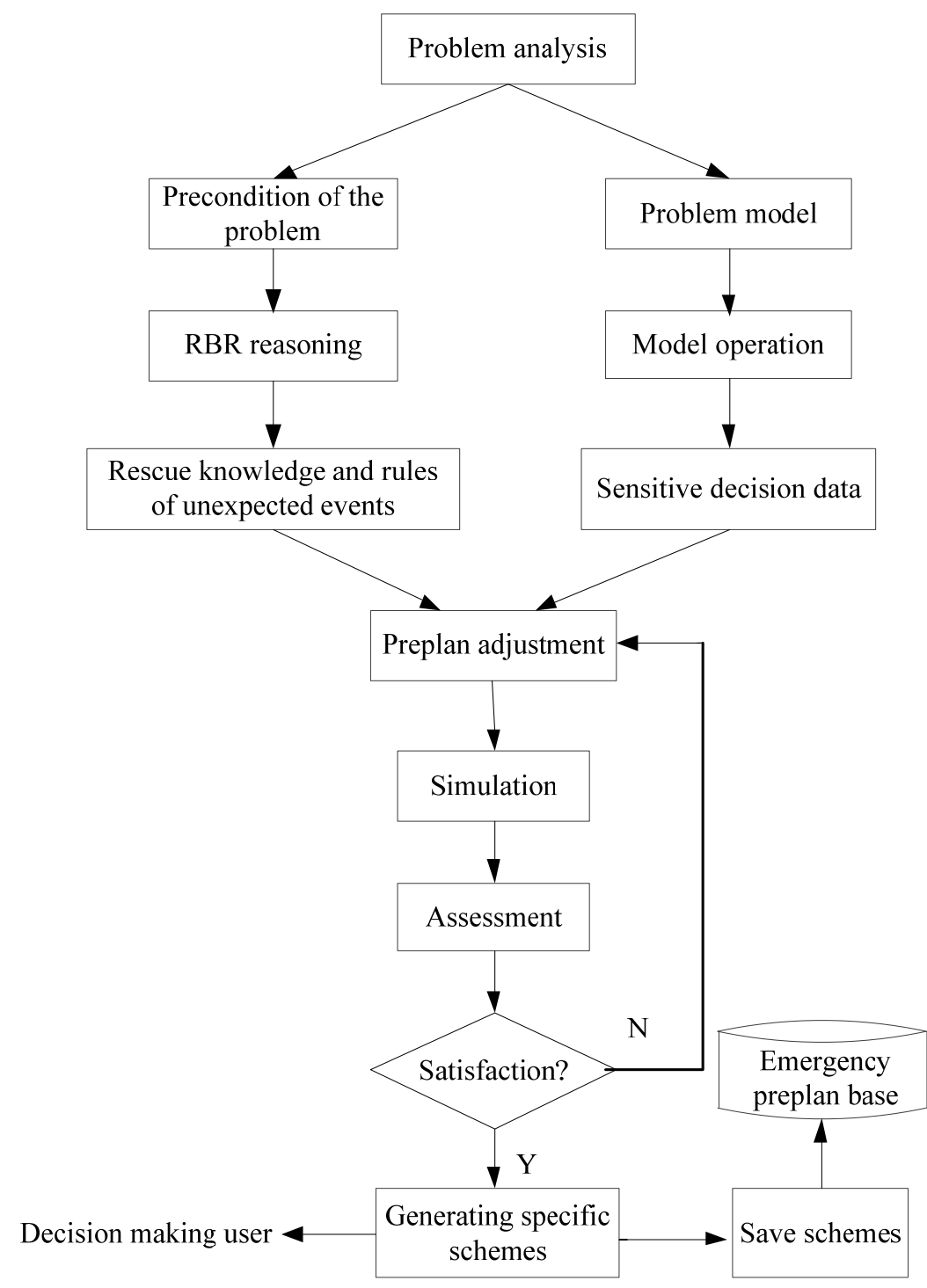

Figure 4. Adjustment process of the preplan

\section{Preplan Learning}

The study here can be seen as a "merger" or "generalization" process. In particular, the learning strategy of a preplan has the following several situations.

Hypothesis source preplan base is $U=\left(X_{1}, X_{2}, \cdots, X_{i}, \cdots, X_{m}\right), i \in[1, m], \mathrm{Xi}$ as a source preplan, the similarity degree of target preplan $\mathrm{Y}$ and $\mathrm{Xi}$ is $\varepsilon_{i} \in[0,1], \eta$ as a threshold defined by an expert 。

(1) $\forall \varepsilon_{i}=0, i \in[1, m]$, the new preplan does not match all the preplans in the preplan base, and at this time, the new preplan can be added to the preplan base.

(2) $\exists \varepsilon_{i}=1, i \in[1, m]$, the new preplan is completely similar to the source preplan, then the new preplan does not join the preplan base.

(3) $\forall \varepsilon_{i}<\eta, i \in[1, m]$, at this time the new preplan can be added to the preplan case.

(4) $\exists \varepsilon_{i}>\eta, i \in[1, m]$, Rewrite the source plan with $\max \left(\varepsilon_{i}\right)$ as a new preplan.

\section{Summary}

The emergency decision support system has important application value when it assists people to make emergency decisions for emergencies. This paper by combining decision support systems and case based reasoning, construct emergency decision support system based on case-based reasoning, 
this is of great significance for improving the ability to respond to emergency decisions, and to enhance the organization's emergency and rapid response in emergencies. In general, case based emergency decision support is a system of learning function, continuous accumulation and continuous improvement, the longer the system is used, the richer the case is, the better the effect of decision support will be.

\section{References}

[1] Zhang Jianhua, Liu Zhongying, 2006. Emergency preplan information system combined with case-based reasoning and rule reasoning. Journal of Tongji University. 30, 7 (July. 2002), 890 894.

[2] Wang Jiyu, Wang Jintao. 2003. Research on emergency decision support system based on case-based reasoning. Management Science. 16, 6(June. 2003), 46 51.

[3] Zhang Rongmei, Tu yanxu. 2002. Intelligent decision support system for traffic accident processing based on CBR. Computer Engineering and Application. 26,10 (Oct. 2002), 247 249.

[4] Li Qihao, Zhao Weidong, Du Xuehan. 2002. A case of medical diagnosis support system based on. Computer Engineering and Application. 18, 12 (Dec. 2002), 173 176.

[5] Liu Yigang. 2001. Research on fast intelligent decision support system based on preplan 